\title{
Nobel Metallic Nanoparticles with Novel Biomedical Properties
}

\section{F Stanley Rosarin and S Mirunalini*}

Department of Biochemistry and Biotechnology, Annamalai University, Annamalainagar - 608002 , Tamil Nadu, India

\begin{abstract}
The strong optical absorption and scattering of noble metal nanoparticles is due to an effect called localized Surface Plasmon Resonance, which enables the development of novel biomedical applications. AuNPs are the most stable metal nanoparticles, and they present fascinating aspects such as their assembly of multiple types involving materials science, the behavior of the individual particles, size-related electronic, magnetic and optical properties [quantum size effect], and their applications to catalysis and biology likewise, AgNPs due to its excellent biocompatibility and antibacterial property raise considerable interest as nanoparticles for biomedical applications. Since the size, shape and composition of silver nanoparticles can have significant effect on their efficacy extensive research has gone into synthesizing and characterizing silver nanoparticles. This article reviews the various synthetic routes currently used for developing stable silver nanoparticles and their biomedical applications in the areas of microbial resistance, antioxidant activity, anticancerous, drug delivery, antiplatlet, antiangiogenic activity, surface enhanced Raman scattering and metal enhanced fluorescence.
\end{abstract}

Keywords: Silver nanoparticles; Gold nanoparticles; Biomedical applications

\section{Introduction}

Nanotechnology deals with synthesis of nanoparticles of various sizes, shapes, chemical compositions and controlled dispersity and their potential use for human benefits [1]. Generally in nanotechnology a nanoparticle as an object which is in small size is defined $10-9 \mathrm{~m}$ that behaves a whole unit in terms of its transport and properties [2]. Nanotechnology is applied to various fields such as physical, chemical, biological and engineering sciences where novel techniques are being developed to probe and manipulate single atoms and molecules. Among all nanoparticles the metallic nanoparticles have applications in diverse areas such as electronics, cosmetics, coatings, packaging and biotechnology. Metallic nanoparticles can be attached to single strands of DNA nondestructively. This opens up avenues for medical diagnostic applications [3]. Nanoparticles can traverse through the vasculature and localize any target organ, this leads to novel therapeutic, imaging and biomedical applications [2]. Ag Nanoparticles and Au Nanoparticles are mostly used in application research in nanotechnology. Previously nanoparticles were produced only by physical and chemical methods with various techniques [4]. Biosynthesis methods have started being used later. In biosynthesis method bacterium, fungi and plant parts are used, this biosynthesis method is ecofriendly comparing to physical and chemical methods. Silver nanoparticles have diverse properties like catalysis, magnetic and optical polarizability, electrical conductivity, antimicrobial activity and Surface Plasmon resonance. Similarly Gold [Au] Nanoparticles have optical, electronic, catalytic, biocompatible properties and potentially high surface reactivity.

\section{Silver nanoparticles and its types}

Silver colloids: In silver nanoparticles if the product is clear then it is ionic silver, not a true silver colloid. Meso silver is called as purest colloidal silver and silver nanoparticles come to the market in 5 types according to its purpose.

Nano xact: Spherical silver formulations are precisely manufactured unaggregated spherical nanoparticles suspended in water.

Biopure silver: It is a high concentration formulation that has been extensively purified to remove residual reactants.

OECD-silver: This silver was given a Organization for Economic Cooperation and Development guidance. Its formulation has been selected as nanotoxicology standards with PVP and citrate surfaces.
Custom silver: This can be produced with custom concentrations, charge, biofunctionalization shells and suspension media.

Silver nanoparticles: They have high optical efficiencies and can be tuned to interact with wavelength ranging from $550 \mathrm{~nm}$ to $950 \mathrm{~nm}$. [www.nanocomposix.com] (Figure 1).

\section{Gold nanoparticles and its types}

There are many subtypes of gold nanoparticles based on the size, shape and physical properties. Gold nanospheres: Gold nanospheres although not exactly spherical in a strict sense [also known as gold colloids] of $2 \mathrm{~nm}$ to over $100 \mathrm{~nm}$ in diameter can be synthesized by controlled reduction of an aqueous Haucl4 solution using different reducing agents under varying conditions. Most commonly citrate is used as a reducing agent, which can produce nearly monodisperse gold nanospheres [5]. SERS [Surface-enhanced Raman scattering] is an optical technique that offers many advantages over traditional

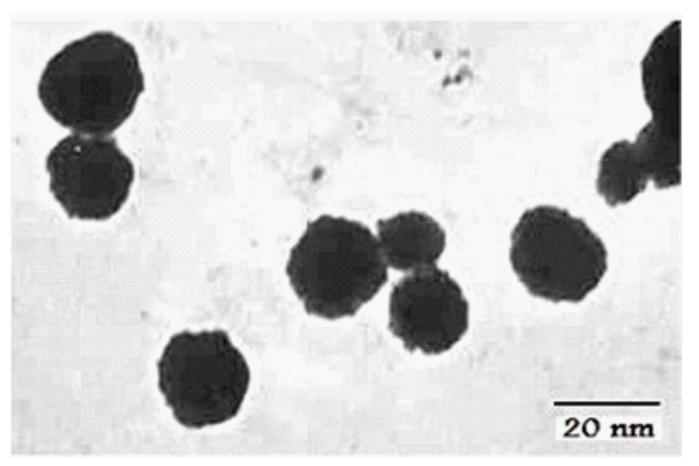

Figure 1: TEM image of $\mathrm{Ag}$ nano particles.

*Corresponding author: Dr. S. Mirunalini, Department of Biochemistry and Biotechnology, Annamalai University, Annamalainagar - 608 002, Tamil Nadu, India, E-mail: mirunasankar@gmail.com

Received May 25, 2011; Accepted June 21, 2011; Published June 28, 2011

Citation: Rosarin FS, Mirunalini S (2011) Nobel Metallic Nanoparticles with Novel Biomedical Properties. J Bioanal Biomed 3: 085-091. doi:10.4172/1948593X.1000049

Copyright: (c) 2011 Rosarin FS, et al. This is an open-access article distributed under the terms of the Creative Commons Attribution License, which permits unrestricted use, distribution, and reproduction in any medium, provided the original author and source are credited. 
Citation: Rosarin FS, Mirunalini S (2011) Nobel Metallic Nanoparticles with Novel Biomedical Properties. J Bioanal Biomed 3: 085-091. doi:10.4172/1948-593X.1000049

technologies, such as fluorescence and chemiluminescence, including better sensitivity, high levels of multiplexing, robustness and superior performance in blood and other biological materials. Gold nanospheres are used as SERS nanoparticles [13 $\mathrm{nm}$ in diameter]. Now gold nanospheres are modified with Cy3-labelled, alkylthiol-capped oligonucleotide strands were used as a probe to monitor the presence of specified target DNA strands [6].

\section{Gold nanorods}

The synthesis of gold nanoparticles has been reported using a wide variety of strategies. Gold nanorods are typically synthesized using the template method, based on the electrochemical deposition of gold within the pores of nanoporous polycarbonate or alumina template membranes [7].

\section{Gold nanoshells}

It is used in optical imaging. Gold nanoshells with surface Plasmon resonance [SPR] peaks in the NIR [Near Infrared Region] can be prepared by coating silica or polymer beads with Gold nanoshells of variable thickness [6](Figure 2).

\section{Physicochemical properties of Au and Agnps}

Properties of nanoparticles depend on structure and composition and can typically be engineered and composition and can typically be engineered or modified by changing the relative influence of interfacial or interphase properties and the macroscopic bulk properties through the characteristic size or dimension of components and domains. The principal parameters of nanoparticles are their shape [including aspect ratios where appropriate], size and the morphological sub-structure of the substrate. Nano particles produce on intense absorption when they excited with an electromagnetic field. That intense absorption attributed to the collective oscillation of electrons on the particle surface termed as a Plasmon resonance. The resonant frequency is highly dependent on particle size. Shape material environment by altering these characteristics the frequency can be shifted over a wide range of wave lengths. Making nano-particles into functional materials for many applications [8]. Silver nanoparticles have diverse properties like catalysis, magnetic and optical polarizability, electrical conductivity,

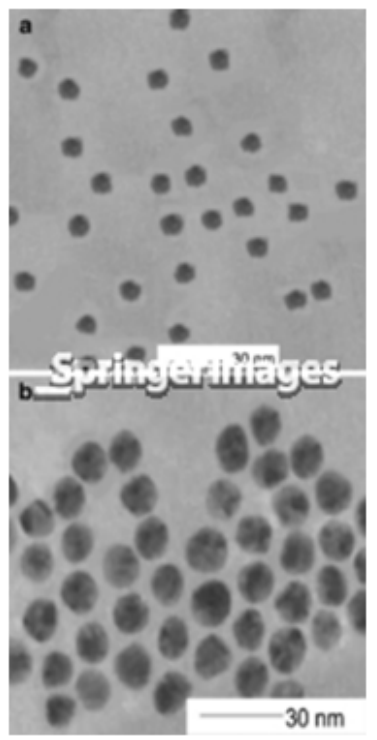

Figure 2: TEM image of Au nanoparticles. and microbial activity and enhanced Raman scattering. Silver NPs have unique optical properties because they support surface plasmons. At specific wavelengths of light the surface plasmons are driven into resonance and the AgNPs have a distinct color that is a function of their size, shape and environment [9]. The plasmons resonance of AgNPs is responsible for yellow color in solution. Any visible change to the NPs in solution typically indicates that the aggregation of the nanoparticles has changed [10]. The wavelength of silver nanoparticles is $430 \mathrm{~nm}$ and is an evidence for the formation of silver nanoparticles.

Silver nanoparticles have many toxicological properties. AgNPs interacts and instigates toxicity in mammalian cancer cells. Cytotoxicity was induced by SNPs [AgNPs] and the role that oxidative stress plays in this process were found in hepatoma cells [11]. Ag+ ions and PVP coated AgNPs induce apoptosis and necrosis in dose dependent manner [12]. Silver nanoparticles are extraordinarily efficient at absorbing and scattering light and, many dyes and pigments, have a colour that depends upon the size and shape of the particle. A unique property of spherical silver nanoparticles is that this SPR peak wavelength can be tuned from $400 \mathrm{~nm}$ [violet length] to $530 \mathrm{~nm}$ [green light] by changing the particle size and the local refractive index near the particle surface [13]. When silver nanoparticles are in solution molecules associate with the nanoparticle surface to establish a double layer of charge that stabilizes the particles and prevents aggregation, and silver nanoparticles in catalysis, magnetic, and optical polarizability, electrical conductivity, antimicrobial activity [14].

Gold nanoparticles [GNPs] as a class of nanomaterials with many unique properties such as colorimetric, conductivity, and non linear optical properties, have been explored for potential applications in biomolecular detection [15]. Gold nanoparticles are precious metal and they have high surface area, easy fictionalization, high electric conductivity, high stability and corrosion resistance and their pronounced plasmon resonance band in the visible range as well as sensitivity to aggregation are amongst their most attractive features [16]. Gold nanoparticles have property of converting allowed radiofrequency into high frequency is known as 'Quantum Dot' property and can be used for destroying cancer cells [17]. Gold is known to be a good electrical and thermal conductor and it is relatively easy for these particles to bind with antibodies, carbohydrates and other pharmacological agents [18]. Nano particles produce on intense absorption when they excited with an electromagnetic field. That intense absorption attributed to the collective oscillation of electrons on the particle surface termed as a plasmon resonance [19]. The resonant frequency is highly dependent on particle size. Shape material environment by altering these characteristics the frequency can be shifted over a wide range of wave lengths. Making nanoparticles into functional materials for many applications [20].

\section{Purpose of Au nanoparticles and Ag nanoparticles}

Delivery: AuNPs can serve as carriers for drug to and gene delivery. Biologically active molecules absorbed on the surfaces can be guided inside cells and released DNA delivery for instane cells and released DNA delivery the basis for gene therapy [21]. Heating AuNPs have a strong light absorbing property that makes AuNPs suitable as heat mediating objects: the absorbed light energy is dissipated into the particles. Surroundings, generating an elevated temperature in their vicinity. This effect can because to open polymer microcapsules, for eg, for drug delivery purposes [22].

Hypothermal therapy: Hypothermal therapy is given to the particle loaded tissue in order ot destruct the malignant cells [23]. 
Sensors: AuNPs can also be used as sensors their optical properties can change upon binding to certain molecules, allowing the detection and quantification of analytes [24]. For labeling certain properties of the are exploited to generate contrast for example in TEM the strong electron absorbing properties of gold nanoparticles make them suitable as a stain for samples with poor contrast such as tissue sample and in immunostaining method they are labeled with antibodies [25]. That shows the small size and the possibility of functionalizing the particles and provides extremely high spatial resolution and specificity in many labeling applications AgNPs. Silver NPs in needed in terms of their interactions with cellular receptors extra - intra cellular proteins, organelles and DNA [26].

Silver nanoparticles of only [1-10 nm] undergo a size dependent interaction with HIV-1 Ag compounds have also been in the medical field to treat the burns and a variety of infections [27]. Salts of silver and their derivatives are commercially employed as antimicrobial agents. Nanoparticles of silver have thus been studied as medium for antibiotic delivery. Metallic nanoparticles exhibit size and shapedependent properties that are of interest for applications ranging from catalysts and sensing to optics, antibacterial activity and data storage. Nanoparticles of silver have thus been studied as a medium for antibiotic delivery and to synthesize composites for use as disinfecting filters and coating materials [28].

\section{Synthesis of Au/Ag NPs}

Previously nanoparticles were produced only by physical and chemical methods some of the commonly used physical and chemical methods are ion sputtering solvothermal synthesis, reduction and sol gel technique. Basically there are two approaches for nanoparticle synthesis. They are bottom up approach and the top down approach. In the top down approach scientist try to formulate nanoparticles using larger ones to direct their assembly [29]. But in the bottom up approach is a process that builds towards larger and more complex systems by starting at the molecular level maintaining precise control of molecular structure [30]. The bottom up approach promises a better change to obtain nanostructures with less defects. The nanoparticle will have more homogenous chemical composition and better short and long range ordering, because this approach is mainly driven by the reduction of Gibb's free energy. Some of the commonly used physical and chemical methods are

1. Chemical reduction: This is carried out by the reduction of an ionic salt in an appropriate medium in the presence of surfactant using reducing agents such as sodium citrate [31].

2. Solvothermal synthesis: This is a versatile low temperature route in which polar solvents under pressure and at temperatures above their building points are used. Under solvothermal conditions, the solubility of reactants increases significantly, enabling reaction to take place at lower temperature [32].

3. Sol gel technique: It is a wet chemical technique used for the fabrication of metal oxides from a chemical solution which acts for integrated network (gel) of discrete particles or polymers. The precursor sol can be either deposited on the substrate to form a film, caste into a suitable container with desired shape or used to synthesize powders [33].

4. Laser ablation: This is the process of removing materials from a solid surface by irradiating with a laser beam. Thus they produce nanoparticles [34].

5. Biosynthesis: Biosynthesis of nanoparticles is a kind of bottom up approach where the main reaction occurring is reduction/ oxidation. Micro organisms and plant extracts are used in general. The advantages of this summarize are 1) Eco friendly 2) They reduces agents employed 3 ) capping agent in the reaction in synthesis by Bacteria many bacteria species had been used when these species were exposed to silver nitrate $\left(\mathrm{AgNO}_{3}\right)$. It produced AgNPs of microbial synthesis of gold nanoparticles was achieved by [35] using mesophilic bacterium shewanella algae with $\mathrm{H}_{2}$ as the electron donor by using fungi AgNPs were synthesized by bioreduction of $\mathrm{AgNo}_{3}$ followed by stabilization and / or encapsulation of the same by a capping agent. Ag and $\mathrm{Au}$ nanoparticles were synthesized by the reduction of $\mathrm{Ag}+$ and $\mathrm{Aucl}_{4}$ ions using extract from (Emblica officinalis). Here the metal ions reduction was motivated by the possibility of inducing shape control of nanoparticles due to the presence of polyphenols in the fruit extract naturally [36](Table 1).

\section{Characterization}

\section{UV visible spectrophotometer}

A beam of light from a visible or UV light or NIR source is separated into its component wavelengths by a prism or diffraction grating. Each monochromatic (Single wave length) beam in turn is split into two equal intensity beams by a half - mirrored device. One is sample beam that passes through the solution and the other beam is a reference beam that passes through the identical cuvet contains solvent and intensities of these light beams are then measured by electronic detectors and compared.

\section{Scanning electron microscopy (SEM)}

Scanning electron microscopy is the microscope that uses electrons instead of light to form an image the resolution of SEM can approach a few $\mathrm{mm}$ and it can operate at magnifications that are easily adjusted from about $10 \mathrm{x}-300,000 \mathrm{xs}$. SEM uses electro magnets rather than lenses; the researcher has more control in the degree of magnification.

\section{$\mathrm{X}$-ray diffraction (XRD)}

It involves the monitoring of the diffraction patterns of x-rays after they interact with the sample. It is used to identify crystal structure.

\begin{tabular}{|c|c|}
\hline Source Used & Nanoparticles synthesized \\
\hline Bacillus subtilis [Bacterium] & Gold [37] \\
\hline Shewanella algae [Algae] & Gold [38] \\
\hline Pseudomonas stutzeri [Bacterium] & Silver [39] \\
\hline Lactobacillus [Bacterium] & Gold, silver, Au-Ag alloy [40] \\
\hline Thermomonospora sp. & Gold [41] \\
\hline [Bacterium] & Gold [42] \\
\hline Rhodococcus [Bacterium] & Gold [43] \\
\hline Chlorella vulgaris [Algae] & Silver [44] \\
\hline MKY3 [Yeast] & Gold, silver [45] \\
\hline Verticillium [Fungi] & zirconia [46] \\
\hline Fusarium oxysporum [Fungi] & Gold, silver, Au-Ag alloy, cadmium, sulfide, \\
\hline Colletotrichum sp. [Fungi] & Gold [47] \\
\hline Aloe vera [Plant] & Silver and gold [48] gold [36] \\
\hline Emblica officinalis [Plant] & Silver, gold and Ag \\
\hline Azadirachta indica [Plant] & Core Au - shell [49] \\
\hline
\end{tabular}

Table 1: Biological synthesis of gold and silver nanoparticles. 


\section{Applications}

\section{Antimicrobial activity of AgNPs}

Silver nanoparticles have antimicrobial activity. It is used as a antimicrobial agent. Colloidal silver is a proven killer of bacteria. It is far more efficient antibiotic than any allopathic pharmaceutical. Colloidal silver is effective in Killing 650 bacteria in less than five minutes, at most in concentrations of five to six parts per million (ppm)

The ASAP nanosilver product has proven effective at eliminating malaria from the blood stream in 4 different preliminary studies, in an overage of just 3.34 days by internal human ingestion. The produced (ASAP nanosilver) was proven in an animal model study at a U.S government NIH lab, to be able to increase the survival rate of mice by $100 \%$ against the dead by H5N1 bird flu virus when using the product orally on a daily basis (relation in peer review journal). The product has been proven to be able to increase the t-lymphocyte count in 7 human AIDS patients by an average of almost $40 \%$ in four months of oral usage at just 2 ounces daily. The product technology is patented to be able to kill TB, MRSA, Hepatitis b, HIV, yeast and too many other pathogens to list here. The point is that it is very broad spectrum antimicrobial agent, which also has been tested found to be completely non-toxic to humans and animals at any level tested. Silver nanoparticles undergo a size dependent interaction with HIV-1. NPs ranging in size from 1 to $10 \mathrm{~nm}$ readily interact with the HIV-1 via preferential binding to gp 120 glycoprotein knobs. This interaction of AgNP, inhibits the virus from binding host cells. Thus they control HIV infection. Colloidal silver is also an anti-parasitic it works by attacking bacteria when they are in their egg-producing stages. Colloidal silver NPs bind with the cell walls of single - cell organisms and inhibit their respiration. Colloidal SNPs a powerful antiviral colloidal silver kills a broad spectrum if viruses. It hills even most eared and dead by viruses, which are not able to be killed by allopathic antiviral and other treatments. It hills HIV, strains of Hepatitis and even emerging strains of flu-viruses. Health Benefits of Colloidal Silver (Angela Kaelin, eHow Contributor, eHow.com).

AgNPs also find application in topical ointment and creams used to prevent infection in burns and open wounds anticancer particles with paclitaxel inhibits the growth of hep G2 cell more effectively AgNps was found as nontoxic on live cells, induced apoptosis on cancerous HT29 [50]. It infers mitochondrial membrane damage during cell apoptosis and fragmentation of BrdU labeled cellular DNA was quantifies by ELISA [51]. DNA breakage was found when cells were treated with AgNPs by using comet assay. Cell cycle arrest raise with AgNPs and mitochondrial dysfunction. Induction of ROS where found these is all the evidences that AgNPs has cytotoxicity and genotoxicity on cells [52]. Gold nanoparticles have been sued in treating B-chronic lymphocytic leukemia (CLL). CLL is an incurable disease predominating characterized by Apoptosis resistance anti VEGF ab has been used previously to treat CLL, however treatment was found more effective when VCGF Ab was attach with $\mathrm{A}_{\mathrm{U}} \mathrm{NPs}$ (1-) chromosomal breakage has been found when cells are treated with AgNPs using cytokines - blocked micronucleus Assay (CBMN) [53].

Anti EGFR antibody - conjugated nonorods binds specifically to the surface of the malignant type cells with a much higher affinity due to over expressed AgNPs EGFR on the cytoplasmic membrane ant cancer of the malignant cells and kills the effect malignant cells with its laser energy. Thus both efficient cancer cell diagnostics and selective photothermal therapy are realized at the same time [54].

\section{Cell imaging}

Aptamer based silver nanoparticles are used in intracellular protein imaging and single nanoparticle spectral analysis, Here AgNPs acts as an illumiophore and the aptamer as a biomolecule specific recognition unit respectively [55]. Gold nanoparticle has strong electric fields at the surface. The absorption and scattering of electro magnetic radiation by noble metal nanoparticles are strongly enhanced. These unique properties provide the potential of designing novel optically active reagents for simultaneous molecular imaging and photothermal cancer therapy. In in vitro studies the gold nanorods are novel contrast agents for both molecular imaging and photo thermal cancer therapy [54] The versatile optical imaging of cells and phantoms with a wide variety of contrast mechanisms. Functional cellular imaging around single molecules has been reported. Functional cellular imaging around single molecules has been reported, tuning advantage of the enhanced second harmonic signal by antibody - conjugated gold nanospheres [56]. Two photon luminescence imaging of cancer ells in a 3D tissue phantom down to the $75 \mathrm{~mm}$ depth has been achieved using gold nanorods. Gold nanorods have been reported for cell imaging using techniques such as dark field light SPR scattering. The use of NIR absorbing gold nanoparticles can significantly enhance the image contrast due to the more substantial differences in optical absorption [hence stronger photo acoustic wave generation] than the endogenous tissue chromophore. Gold NPs are used also in vivo imaging many paramagnetic nanoparticles have been used for preclinically and clinically [57]. Au3Cl1 nanoshells are used to enhance the contrast of blood vessels in vivo, which suggested their potential us in MR angiography as blood - Pool agents.

\section{Biosensors}

Prostate specific Antigen (PSA) has been used to confirm the presence of semen and absence of sperm in sexual assault cases the use of PSA is forensic analysis has a different set of requirements such as lack of sample or need to extract. Nanoparticles and nanostructures are used to enable the analytic detection, gold NPs probes are attached to Antibodies that are labeled with Raman dyes. This assay has been regarded robust for PSA detection due to being label free and in expensive in this method PSA detection is done by enhancement Raman Scattering [58].

\section{Cancer therapy (anticancer)}

Conventional strategies for cancer intervention include surgery, chemotherapy, and radiation therapy.

Taking advantage of their unique properties, most studies of gold nanoparticle - based cancer therapy have used photothermal therapy for the destruction of cancer cells or tumor tissue, which may be potentially useful in the clinical setting. Gold nanoshells are sufficiently large (about 100-300 $\mathrm{nm}$ in diameter) to have SPR peaks in the WIR region. In one pioneering study, human beast carcinoma cells incubated with gold nanoshells were found to undergo photothermally induced morbidity upon exposure to NIR light. In one study $93 \%$ of tumor necrosis and regression. Was observed in a high dose nanoshell $(8.5 \mathrm{ml} / \mathrm{g})$ treated group [59].

\section{Diagnosis}

Gold nanoparticles (GNPs) as a class of nanomaterials with many unique properties such as colorimetric conductivity, and nonlinear optical properties, have been explored for potential applications in biomolecular detection. GNPs can be used to quantitatively detect 
Citation: Rosarin FS, Mirunalini S (2011) Nobel Metallic Nanoparticles with Novel Biomedical Properties. J Bioanal Biomed 3: 085-091. doi:10.4172/1948-593X.1000049

nucleic acids and proteins in clinical samples. For example, a DNA based method for rationally assembling gold nanoparticles into macroscopic materials has been reported by mirkin and coworkers. For protein detection, an aggregation - based immunoassay for antiprotein A using gold nanoparticles has been developed [60]. The hyper-Rayleigh scattering signals of aggregated gold nanoparticles labeled with immunoglobulin $\mathrm{G}$ could be used to quantity antibody / Antigen in aqueous solution, [15]. These two methods could only detect proteins at the microgram level, which limits their applications in immunoassay especially in early cancer diagnosis.

\section{Antioxidant properties}

Previously it was reported that AgNPs reduce mitochondrial function and increase membrane leakage in mammalian germline stemcells and increase reaction oxygen species (ROS) generation, deplete antioxidant GSH content, and reduce mitochondrial function in BRL 3A rat liver cells [61]. In addition to the well known specific effects of peroxide, superoxide and hydroxyl radicals, ROS in general also cause DNA damage, including a multitude of oxidized base lesions, abasic sites, single and double-strand breaks; all of these can be cytotoxic and or mutageni $[62,63]$. Excessive ROS generation is also known to induce apoptosis/cell death in various cell culture models. Excessive production of ROS in the cell is known to induce apoptosis. ROS generation has been shown to play an important role in apoptosis induced by treatment with AgNPs [64]. It has been studied that AgNPs increase the production of intracellular ROS and decrease reduced glutathione levels, leading to apoptosis [65]. Showed that AgNPs decreased GSH levels through the inhibition of GSH levels through the inhibition of GSH synthesizing enzymes (GCLS and GSS), leading to the generation of ROS. However, GSH is not the only intracellular antioxidant defence, therefore it remains to be determined if the other intracellular antioxidant systems 9e.g) superoxide dismutase, catalase are also impaired by AgNPs.

\section{Other applications}

Gold nano particles have received great attention as an antiangrogenic agent with the evidence nanoparticles block VEGF-induced retinal vascular permeability invivo [66]. Ag-NPs to be a potent antiangiogenic molecule that inhibits angiogenesis in VEGF(Vascular endothelial growth factor) -induced BRECs (bovine retinal endothelial cells) through the inhibition of the PI3K/Akt cell-survival signal in a similar pattern of PEDF (Pigment epithelium-derived factor also known as serpin F1 (SERPINF1), is a multifunctional secreted protein that has anti-angiogenic functions) This in turn alters the downstream signaling and subsequent cellular responses. These findings provide strong evidence that nanostructures cannot only passively interact with cells, but also actively engage and mediate the molecular processes that are essential for regulating cell functions. The particles of $50 \mathrm{~nm}$ have been used. Further studies involve a size-dependent study involving AgNPs as anti-angiogenic molecule that can elucidate the potency, efficacy and efficiency compared to PEDF. This study provides a mechanism to account for the inhibition of Ag-NPs on VEGF-mediated angiogenesis and also suggest that Ag-NPs could be the targeted therapeutic molecule for the treatment of diabetic retinopathy and other eyerelated neovascular diseases [67]. Gold nanoparticles have found application in analytical methods such as colorimetric techniques for the determination of heavy metal ions in aqueous solutions gold nano particles also used in the field of sensor [68]. Nanosilver has an innate antiplatelet property and effectively prevents integrinmediated platelet responses both in vivo and in vitro in a concentration dependent manner. It is useful to treat thrombatic disorders. It regulates and maintains platelets in an inactive state treatment by anticoagulant and Thrombatic therapies are usually associated with serious bleeding complication [69]. Forster Resonance energy transfers have served as a molecular ruler that reports conformational changes and intramolecular distances of single bio molecules. This molecular ruler are rigid oligoproline cross linking reagent of defined length such reagents are useful in probing the structures of biological macromolecules insolution, but they suffer from low fluctuating signal intensities and limited observations. It has been demonstrated that plasmon coupling can be used to monitor distances between single pairs of gold and silver nanoparticles dimmers in real time and studied the kinetics of single DNA hybridization events these are known as plasmon rulers [70]. The toxicity of Ag nanoparticles is investigated using a panel of recombinant bioluminescent bacteria. The presence of the nanoparticles leads to the production of a superoxide radical. Furthermore, the Ag nanoparticles damage the cellular membranes, causing a disruption in the ion efflux system. Thus, the cells cannot effectively extrude the $\mathrm{Ag}$ ions and, hence, $\mathrm{Ag}$ nanoparticles cause more damage than do Ag ions [71](Figure 3, Figure 4).

Antioxidant activity

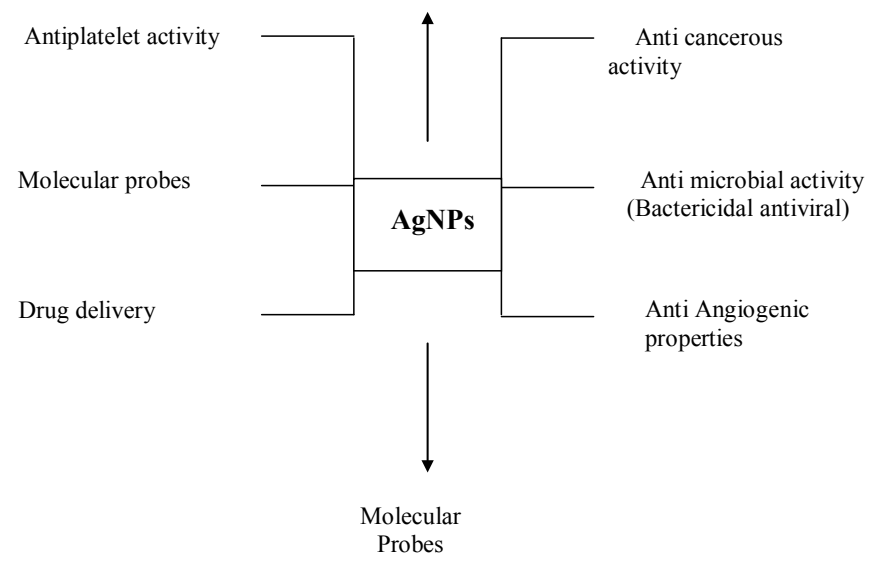

Figure 3: Applications of Ag nanoparticles.

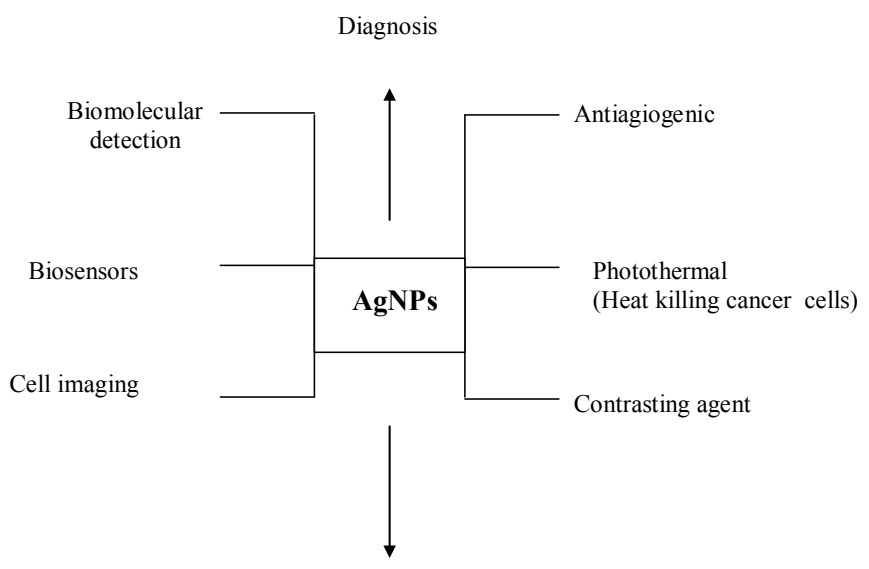

Anti cancerous

Figure 4: Applications of Au nanoparticles. 
Citation: Rosarin FS, Mirunalini S (2011) Nobel Metallic Nanoparticles with Novel Biomedical Properties. J Bioanal Biomed 3: 085-091. doi:10.4172/1948-593X.1000049

\section{Conclusion}

In this review, we have focused on the current applications of AuNPs and AgNPs in biomedical science. In addition, the biocompatibility and photo-optical distinctiveness of AuNPs are now proven to be powerful in diagnostic and biosensing applications. With the "right" combination of delivery agents and particle size, AuNPs-based therapeutics could effectively kill the diseased cells while eliminating the horrendous side effects of the conventional chemotherapeutic agents. Ag nanoparticles shows toxicity to cancerous cells and also on normal cells, but target delivery helps to achieve good results. And it has been proven that AgNPs have antiplatelet activity and anti angiogenic activity. Theses two properties of AgNPs will be helpful in therapeutics. Physical, Chemical and biological methods are used to synthesize Au and Ag nanoparticles. Plants are used to synthesize these metallic nanoparticles and in this plant mediated synthesis, plants are used as a stabilizing agent and capping agent. Plants reduce the ions of these metals and help to produce nanoparticles. This enhances the activity of the $\mathrm{Au}$ and Ag Nanoparticles. Au nanoparticles plays a major role in molecular diagnosis. As the nanoparticle is less than $1 \mu \mathrm{M}$ in diameter, it is useful in diagnosis.

Taken together gold and silver nanoparticles are useful in almost all medical fields in It reaches sensitivities of a few molecules, perhaps even a single molecule, per nanoparticle sensor element. Gold and silver nanoparticles were employed as Glucose sensing platforms through the modification of their surfaces with dextran and boronic acid groups, respectively. This concludes that gold and silver nanoparticles have novelty as biosensors in molecular level. Photo thermal therapy is the unique property of gold nanoparticles and silver nanoparticles are known for antimicrobial activity.

\section{References}

1. Vineet K, Sudesh KY (2009) Plant mediated synthesis of silver and gold nanoparticles and their applications. J Chem Technol Biotechnol 84: 151-157.

2. Singh MS, Manikandan, Kumaraguru AK (2010) Nanoparticles: A New Technology with Wide Applications. Res J Nanosci Nanotechnol 10: 19962014.

3. Thakkar KN, Mhatre SS, Parikh RY (2000) Biological synthesis of metallic nanoparticles. Nanomedicine 6: 257-262.

4. Tripathi A, Chandrasekaran N, Raichur AM, Mukherjee A (2009) Process variables in biomimetic synthesis of silver nanoparticles by aqueous extract of Azadirachta indica (Neem) leaves. J Biomed Nanotechnol 5: 93-98.

5. Hiramatsu H, Frank E, Osterloh (2004) A Simple Large-Scale Synthesis of Nearly Monodisperse Gold and Silver Nanoparticles with Adjustable Sizes and with Exchangeable Surfactants. Chem Mater 16: 2509-2511.

6. Cai W, Gao T, Hong H, Sun J (2008) Applications of gold nanoparticles in cancer nanotechnology. Nanotechnology, Science and Applications 1: 17-32.

7. Huczko A (2000) Template-based synthesis of nanomaterials. Applied Physics A: Materials Science \& Processing 70: 4.

8. Otsuka C, Nagasaki Y, Kataoka K(2003) PEGylated nanoparticles for biological and pharmaceutical applications. Adv Drug Deliv Rev 55: 403-419.

9. Noguez C (2007) Surface Plasmons on Metal Nanoparticles: The Influence of Shape and Physical Environment. J Phys Chem C 111: 3806-3819.

10. Pietrobon B, Kitaev V (2008) Photochemical Synthesis of Monodisperse SizeControlled Silver Decahedral Nanoparticles and Their Remarkable Optical Properties. Chem Mater 20: 5186-5190.

11. Kim S, Choi JE, Choi J, Chung KH, Park K, et al. (2009) Oxidative stressdependent toxicity of silver nanoparticles in human hepatoma cells. Toxicol invitro 23: $1076-1084$

12. Foldbjerg R, Olesen P, Hougaard M, Dang DA, Hoffmann HJ, et al. (2009) PVPcoated silver nanoparticles and silver ions induce reactive oxygen species, apoptosis and necrosis in THP-1 monocytes. Toxicol Lett 190: 156-162.
13. Ma H, Jiao Y, Yin B, Wang S, Zhao S, et al. (2004) Spontaneous Organization of Individual Silver Nanoparticles into One-Dimensionally Ordered Nanostructures. ChemPhysChem 5: 713-716.

14. Shipway AN, Katz E, Wilner I (2000) Nanoparticle arrays on surfaces for electronic, optical, and sensor applications. ChemPhyschem 1: 18-52.

15. Zhang CX, Zhang Y, Wang X, Tang ZM, Lu ZH (2003) Hyper-Rayleigh scattering of protein-modified gold nanoparticles. Anal Biochem 320: 136-140.

16. Gun J, Rizkov D, Lev O (2009) Oxygen plasma-treated gold nanoparticlebased field-effect devices as transducer structures for bio-chemical sensing Micro Chim Acta 164: 395-404

17. Claus P, Brückner A, Mohr C (2000) Supported Gold Nanoparticles from Quantum Dot to Mesoscopic Size Scale: Effect of Electronic and Structural Properties on Catalytic Hydrogenation of Conjugated Functional Groups. J Am Chem Soc 122: 11430-11439.

18. Baptista P, Pereira E, Eaton P, Doria G, Miranda A, et al. (2008) Gold nanoparticles for the development of clinical diagnosis methods. Anal Bioanal Chem 391: 943-950.

19. Jensen TR, Malinsky MD, Christy L (2000) Nanosphere Lithography: Tunable Localized Surface Plasmon Resonance Spectra of Silver Nanoparticles. J Phys Chem B 104: 10549-10556.

20. Jain PK, Huang X, El-Sayed IH, El-Sayed MA (2007) Review of Some Interesting Surface Plasmon Resonance-enhanced Properties of Noble Metal Nanoparticles and Their Applications to Biosystems. Plasmonics 2: 107-118.

21. Han G, Ghosh P, Rotello VM (2007) Special Focus: Advances in Nanomedicine Symposium - Review: Functionalized gold nanoparticles for drug delivery. Nanomedicine 2: 113-123.

22. Gaiduk A, Ruijgrok PV, Yorulmaz M, Oritt M (2011) Making gold nanoparticles fluorescent for simultaneous absorption and fluorescence detection on the single particle level. Phys Chem Chem Phys 13: 149-153.

23. Huang X, Mostafa A, Sayed E (2010) Gold nanoparticles: Optical properties and implementations in cancer diagnosis and photo thermal therapy. Journal of Advanced Research 1: 74-86.

24. Jain KK (2005) Nanotechnology in clinical laboratory diagnostics. Clinica Chimica Acta 358: 37-54.

25. El-Sayed IH, Huang X, El-Sayed MA (2005) Surface Plasmon Resonance Scattering and Absorption of anti-EGFR Antibody Conjugated Gold Nanoparticles in Cancer Diagnostics: Applications in Oral Cancer. Nano Lett 5: $829-834$

26. Simkó M, Fiedeler U, Gazsó A (2011) The impact of nanoparticles on cellular functions nanotrust dossiers. No 007 January 2011.

27. Elechiguerra JL, Burt JL, Morones JR, Camacho-Bragado A, Gao X, et al. (2005) Interaction of silver nanoparticles with HIV-1. J Nanobiotechnology 3: 6

28. Ghoush S, Ashutosh upadhay, Abhishek KR (2010) Investigation of Antimicrobial activity of silver nanoparticle loaded cotton fabrics which may promote wound healing. International Journal of Pharma and Biosciences 1: 56-62.

29. Acosta $E$ (2009) Bioavailability of nanoparticles in nutrient and nutraceutical delivery. Curr Opin Coll Int Sci 14: 3-15.

30. Lee SW, Chang WJ, Bashir R (2007) "Bottom-up" approach for implementing nano/microstructure using biological and chemical interactions. Biotechnol and Bioproc Eng. 12: 185-199.

31. Wang H, Qiao X, Chen J (2005) Preparation of silver nanoparticles by chemical reduction method. Colloids and Surfaces A: Physicochemical and Engineering Aspects 256: 111-115.

32. Yang Y, Matsubara S, Xiong L (2007) Solvothermal Synthesis of Multiple Shapes of Silver Nanoparticles and Their SERS Properties. J Phys Chem 111 9095-9104.

33. Jia JJ, Wang B, Wu A (2002) A Method to Construct a Third-Generation Horseradish Peroxidase Biosensor: Self-Assembling Gold Nanoparticles to Three-Dimensional Sol-Gel Network. Anal Chem 74: 2217-2223.

34. Besner S, Kabashin AV, Meunier M (2007) Two-step femtosecond laser ablation-based method for the synthesis of stable and ultra-pure gold nanoparticles in water. Appl Phys A 88: 269-272. 
Citation: Rosarin FS, Mirunalini S (2011) Nobel Metallic Nanoparticles with Novel Biomedical Properties. J Bioanal Biomed 3: 085-091. doi:10.4172/1948-593X.1000049

35. Konishi Y, Ohno K, Saitoh N (2004) Microbial synthesis of gold nanoparticles by metal reducing bacterium. Trans Mater Res Soc Jpn 29: 2341-2343.

36. Ankamwar B, Damle C, Ahmed A, Sastry M (2005) Biosynthesis of gold and silver nanoparticles using emblica officinalis fruit extract their phase transfer and transmetallation in an organic solution. J Nanosci Nanotechnol 5: 16651671.

37. Beveridge TJ, Murray RG (1980) Site of metal deposition in the cell wall of Bacillus subtilis. J Bacteriol 141: 876-887.

38. Konishi Y, Nomura T, Tsukiyama T (2004) Microbial preparation of gold nanoparticles by anaerobic bacterium. Trans Mater Res Soc Jpn 29: $2341-$ 2343.

39. Slawson RM, Van Dyke MI, Lee H, Trevor JT (1992) Germanium and silver resistance, accumulation and toxicity in microorganisms. Plasmid 27: 72-79.

40. Nair B, Pradeep T (2002) Coalescence of nanoclusters and formation of submicron crystallites assisted by Lactobacillus strains. Cryst Growth Des 2 293-298.

41. Ahmad A, Senapati S, Khan MI, Kumar R, Sastry M, et al. (2003) Extracellular biosynthesis of monodisperse gold nanoparticles by a novel extremophilic actinomycete, Thermomonospora sp. Langmuir 19: 3550-3553.

42. Ahmad A, Senapati S, Khan MI, Ramani R, Srinivas V, Sastry M, et al (2003) Intracellular synthesis of gold nanoparticles by a novel alkalotolerant actinomycete, Rhodococcus species. Nanotechnology 14: 7.

43. Hosea M, Greene B, Mcpherson R (2001) Accumulation of elemental gold on the alga Chlorella vulgaris. Inorg Chim Acta 123: 161-165.

44. Kowshik M, Ashtaputre S, Kharrazi S (2003) KM Extracellular synthesis of silver nanoparticles by a silver-tolerant yeast strain MKY3. Nanotechnology 14 : 95-100.

45. Mukherjee P, Ahmad A, Mandal D, Senapati S, Sainkar SR, et al. (2001) Bioreduction of AuCl4- ions by the fungus, Verticillium sp. and surface trapping of the gold nanoparticles formed. Angew Chem Int Ed Engl 40: 3585-3588.

46. Mukherjee P, Senapati S, Mandal D, Ahmad A, Khan MI, et al. (2002) Extracellular synthesis of gold nanoparticles by the fungus Fusarium oxysporum. Chem bio chem 3: 461-463.

47. Shankar SS, Ahmad A, Parischa R (2003) Bioreduction of chloroaurate ions by geranium leaves and its endophytic fungus yields gold nanoparticles of different shapes. J Mater Chem 13: 1822-1826.

48. Chandran SP, Chaudhary M, Pasricha R, Ahmad A, Sastry M (2006) Synthesis of Gold Nanotriangles and Silver Nanoparticles Using Aloevera Plant Extract. Biotechnol Prog 22: 577-583

49. Shankar SS, Rai A, Ahmad A, Sastry M (2004) Rapid synthesis of Au, Ag, and bimetallic Au core-Ag shell nanoparticles using Neem (Azadirachta indica) lea broth. Journal of Colloid and Interface Science 275: 496-502.

50. Zuco V, Supino R, Righetti SC (2002) Selective cytotoxicity of betulinic acid on tumour cell lines but not on normal cells. Cancer letters 175: 17-25.

51. Gopinath P, Gogoi SK, Chattopadhya A (2003) Implications of silver nanoparticle induced cell apoptosis for in vitro gene therapy. Nanotechnology 19-27.

52. Asharani PV, Kah mun GL, Hande MP (2009) Cytotoxicity and genotoxicity of silver nanoparticles in human cells. ACS nano 13: 13-28.

53. Mukherjee P, Bhattacharya R, Bone N, Lee YK, Patra CR (2007) Potential therapeutic application of gold nanoparticles in B-chronic lymphocytic leukemia (BCLL): enhancing apoptosis. J Nanobiotechnology 8: 5-4.

54. Huang X, El-Sayed IH, Qian W, El-Sayed MA (2006) Cancer Cell Imaging and Photothermal Therapy in the Near-Infrared Region by Using Gold Nanorods. J Am Chem Soc 128: 2115-2120.

55. Chen LQ, Xiao SJ, Peng L, Wu T, Ling J, et al. (2010) Aptamer-Based Silver Nanoparticles Used for Intracellular Protein Imaging and Single Nanoparticle Spectral Analysis. J Phys Chem B 114: 3655-3659.

56. Peleg G, lewis A, Lineal M, Loew LM (2009) Nonlinear optical measurement of membrane potential around single molecules at selected cellular sites. Proc Natl Acad Sci USA 96: 6700-6704.

57. de Roos A, Doornbos J, Baleriaux D, Bloem HL, Falke TH (1986) Clinical applications of gadolinium DTPA in MRI. Magn Reson Annu 113-145.
58. Hwang KS, Lee JH, Park J, Yoon DS, Park JH (2010) In-situ quantitative analysis of a prostate-specific antigen (PSA) using a nanomechanical PZT cantilever. Labchip 4: 547-552.

59. Stern MJ, Stanfield J, Kabbani W, Hsieh JT, Cadeddu JA (2008) Selective Prostate Cancer Thermal Ablation With Laser Activated Gold Nanoshells. J Urol 179: 748-753

60. Thanh NT, Rosenzweig Z (2002) Development of an Aggregation-Based Immunoassay for Anti-Protein A Using Gold Nanoparticles. Anal Chem 74 1624-1628.

61. Braydich-Stolle L, Hussain S, Schlager JJ, Hofmann MC (2005) In Vitro Cytotoxicity of Nanoparticles in Mammalian Germline Stem Cells. Toxicol Sc 88: $412-419$

62. Bhakat KK, Mokkapati SK, Boldogh I, Hazra TK, Mitra S (2006) Acetylation of human-8-oxoguanine-DNA glycosylase by p300 and its role in 8-oxoguanine repair invivo. Mol cell boil 26: 1654-1665.

63. Diakowska D, Lewandowski A, Kopeć W, Diakowski W, Chrzanowska T (2007) Oxidative DNA Damage and total antioxidant status in serum of patients with esophageal squamos cell carcinoma. Hepatogastroenterology 78: 1701-1704.

64. Carlson C, Hussain SM, Schrand AM, Braydich-Stolle LK, Hess KL, et al. (2008) Unique Cellular Interaction of Silver Nanoparticles: Size-Dependent Generation of Reactive Oxygen Species. J Phys Chem B 112: 13608-13619.

65. Piao MJ, Kang AK, Lee IK, Kim HS, Kim S (2011) Silver nanoparticles induce oxidative cell damage in human liver cells through inhibition of reduced glutathione and induction of mitochondria-involved apoptosis. Toxicol Lett 201 $92-100$

66. Mukarjee P, Battacharya R, Wang P (2005) Antiangigenic properties of gold nanoparticles. Clin cancer Res 11: 3530-3534.

67. Gurunathan S (2009) Silver nanoparticles inhibit VEGF-and IL-1 -induced vascular permeability via Src dependent pathway in porcine retinal endothelia cells. Journal of Nanobiotechnology 7-8.

68. Sedeno PY, Pingarron JM (2005) Gold nanoparticle based electrochemical biosensors. Anal bioanal chem 382: 884-886.

69. Shrivastava S, Bera T, Sunil K, Gajendra Singh P, Ramachandrarao, et al. (2009) Characterization of Antiplatelet Properties of Silver Nanoparticles. ACS Nano 3: 1357-1364.

70. Sonnichsen C, Reinhard BM, Liphardt J, Alivisatos AP (2005) A molecular ruler based on Plasmon coupling of single gold and silver nanoparticles. Na Biotechnol 23: 741-745.

71. Hwang ET, Lee JH, Chae YJ, Kim YS, Kim BC, et al. (2008) Analysis of the Toxic Mode of Action of Silver Nanoparticles Using Stress-Specific Bioluminescent Bacteria. Small 4: 746-750. 